\title{
A Facile Autoxidation of an Allylic Alcohol in Air
}

\author{
Fathia A. Mosa ${ }^{a^{*}}$ and Andrew Whiting ${ }^{b}$ \\ aDepartment of Chemistry, Sirte University, Sirte, Libya.

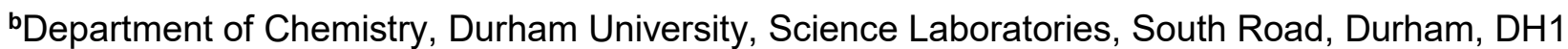 \\ 3LE, UK. \\ Email: *fathia@su.edu.ly
}

Keywords: Autoxidation; Cinnamaldehyde; Alkadienyl alcohol; Benzaldehyde; Decomposition

\begin{abstract}
An (4E,6E)-alkadienyl alcohol which is a solid compound which can be stored at rt, upon dissolving into a suitable solvent undergoes facile autoxidation $(4 E, 6 E)$-alkadienyl alcohol 1 in air at room temperature. The result is complete decompose leading to a mixture of products, including benzaldehyde (24\%) and cinnamaldehyde (29\%). Possible mechanistic explanations for the autoxidation are discussed.
\end{abstract}

\section{Introduction}

The autoxidation (air-induced oxidation) of certain organic compounds is well known, with a number of studies attempts to explain this type of this oxidation.1-4 Generally, the autoxidation of electron-rich substrates under ambient conditions can be characterized by $\alpha$-oxidation (or allylic) oxidation products. ${ }^{2}$ For example, reports that unsaturated "fats" being particularly, susceptible to autoxidation and there being a need for allylic hydrogens to be present, in order to allow reaction with peroxy radicals." 4 Compound 1 was required as model substrate for study developing methodology to tackle the total synthesis of viridenomycin, ${ }^{5}$ however, and as reported in this communication, it turned out to be can susceptible to undergo to facile reaction dioxygen resulting autoxidation.

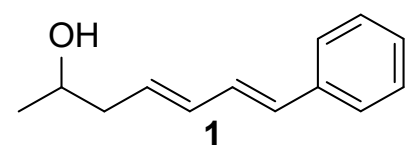

\section{Results and Discussion}

In order to access the racemic allylic alcohol 1, initially a vinylboronate 2-vinyl iodide 3 HeckMizoroki cross-coupling ${ }^{6}$ was carried out as shown in Scheme 1, to access ( $\left.4 E, 6 E\right)$-dienyl boronate ester 4.
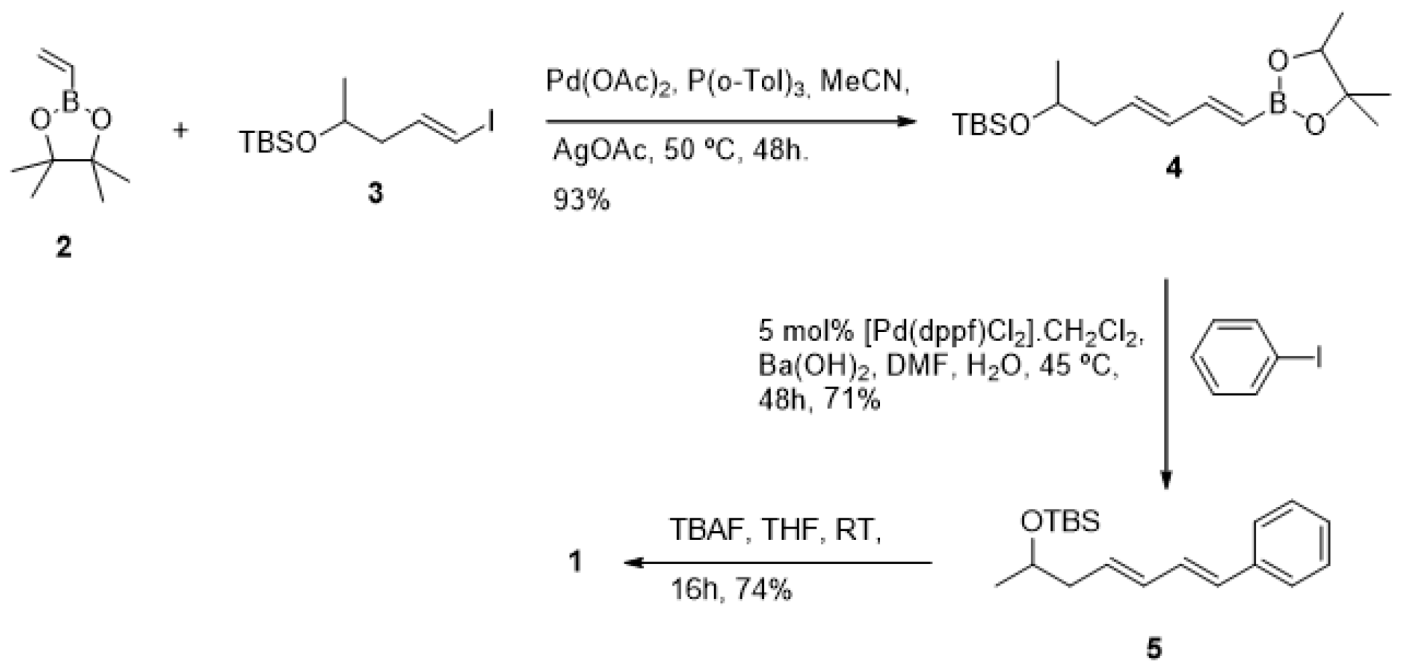

Scheme 1. Synthesis of allylic alcohol 1 
Then, alcohol 1, was synthesized by a palladium-catalyzed coupling reaction between the iodobenzene 1 and boronate ester 3, followed by cleavage of the TBS-group of silyl ether 5 using TBAF (Scheme 1).

The $(4 E, 6 E)$-alkadienyl alcohol 1 was found to be unstable at room temperature even when stored as a solid, and thus was preferably kept in the fridge under an inert atmosphere because it decomposed at $\mathrm{rt}$ in air to other components. Indeed, a solid sample of $(4 E, 6 E)$-alkadienyl alcohol 1 after standing at $\mathrm{rt}$ in air was transformed into an oily material. Purification by silica gel chromatography gives both benzaldehyde (24\%) and cinnamaldehyde (29\%) respectively together with other mixed and unidentified fractions (Equation 1).

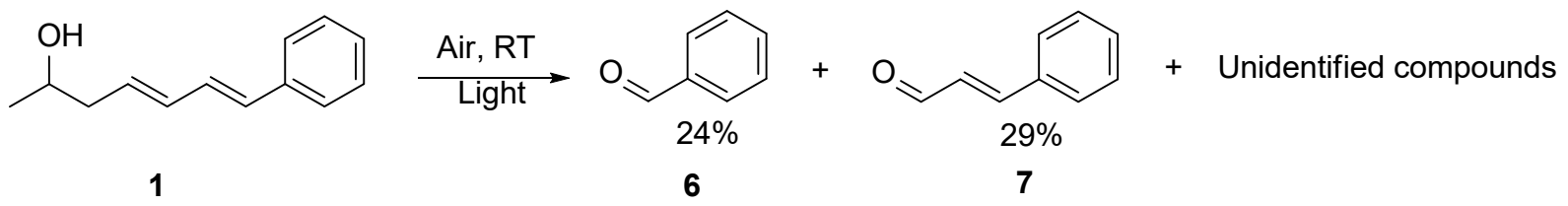

Equation 1. The decomposition of allylic alcohol 1

The interpretation of the decomposition of allylic alcohol 1 in the air can occur through several possible routes and the main reason for such sensitivity to reactivity is possibly that the photo-autoxidate process is likely to involve cleavage of the electron-rich olefins which leads to the decomposition of the whole structure. The autoxidation mechanism has been explained before on the basis of the free-radical processes. ${ }^{1-4}$ Auto-oxygenation of allylic alcohol 1 could therefore occur by precisely such mechanistic steps.

Hence, we suggest the mechanism for the auto-oxidation of 1 (Scheme 2) could be similar to the mechanism of autoxidation of unsaturated fats (linoleate autoxidation). ${ }^{4}$ The first step would be hydrogen abstraction on the reactive allylic carbon of compound 1 by the reaction with molecular oxygen which provides unsaturated hydroperoxide 9 . The next step would be the decomposition of an unsaturated hydroperoxide 8 by the homolytic cleavage of the oxygen-oxygen bond to yield an alkoxy radical 9 and hydroxy radical $(\mathrm{OH}) .{ }^{1 \mathrm{~d}}$ Carbon-carbon cleavages (a) and (b) lead to aldehydes 10 and 7 and olefin radicals 11 and 14, which could then react with hydroxy radicals to form enols 12 and 15, and hence tautomerize to the corresponding aldehydes 13 and 16 (Scheme 2).<smiles>CC(O)/C=C/C(/C=C/c1ccccc1)OOC(C)C(C)O</smiles>

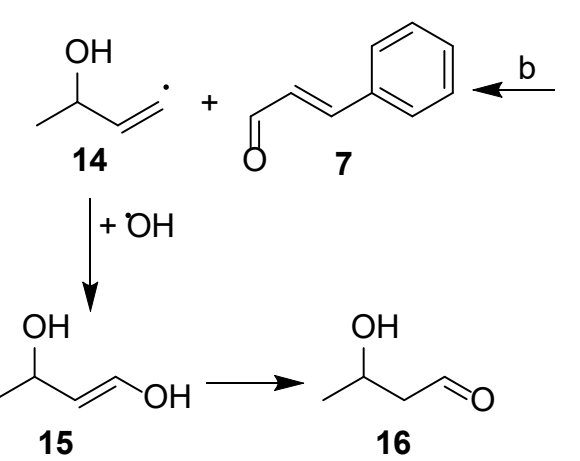<smiles>CC(O)C=CC(O)(O)C=Cc1ccccc1</smiles>

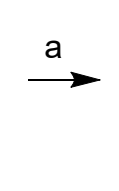<smiles>CC(O)/C=C/[C+]=O</smiles><smiles>Cc1ccccc1C=O</smiles>

Scheme 2. Suggested mechanism for autoxidation of $(4 E, 6 E)$-alkadienyl alcohol 1

The reaction of molecular oxygen with pentadienyl radical 17 can produce a mixture of unsaturated hydroperoxides 18 and 26. The most favoured site for the attack of molecular oxygen is at the ends of pentadienyl radical 17 ( $\mathrm{a}$ and $\mathrm{b}$ ), as shown in Scheme 3. In the following reaction sequence, the first step of the decomposition of the unsaturated hydroperoxides 18 and 26 is 
proposed to be the homolytic cleavage of the oxygen-oxygen bond to yield alkoxy radicals 19 and 27 and hydroxy radicals. ${ }^{4}$ Carbon-carbon cleavages (a) and (b) provides aldehydes 6, 20, 7 and 28, and the free radicals 21, 23, 29 and 32 (Scheme 3). The phenyl radical 21 can react with $\mathrm{OH}$ to give phenol 22 while, the free radical 32 can react with $\mathrm{OH}$ to give a geminal diol 33.<smiles>CC(O)C(C=CC=Cc1ccccc1)OO</smiles><smiles>CC(O)C=CC=Cc1ccccc1</smiles>

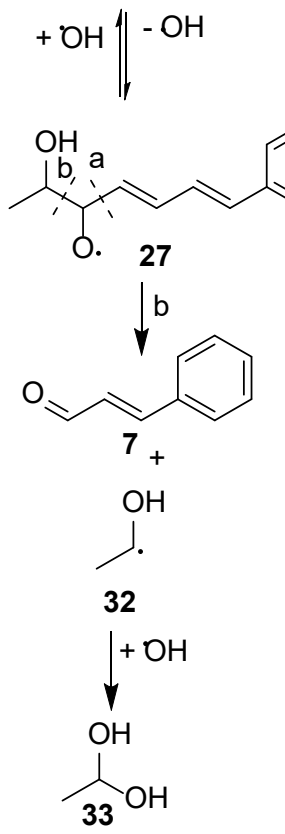<smiles>C=CC=Cc1ccccc1</smiles>
17<smiles>C[C@H](O)/C=C/C=C/C(O[18OH])c1ccccc1</smiles><smiles>C=CC=CC(C)C(C)O</smiles>

Scheme 3. Suggested mechanism for autoxidation of (4E,6E)-alkadienyl alcohol 1

Another suggested mechanism depends on the sensitivity of electron-rich compounds to both air and light (photo-oxidation). The photosensitized alcohol 1 can be the subject of abstract hydrogen by light to give pentadienyl radical 17 (Scheme 4).

In another approach, both unsaturated hydroperoxides 18 and 26 can lose hydrogen to lead to the formation of five-membered epidioxides 35 and 38. ${ }^{4}$ The five-membered ring in compounds 35 and 38 can then be reopened and gain a hydrogen radical to give unsaturated hydroperoxide 8 , which decomposes as explained in Scheme 4. 
<smiles>[R6]OC(/C=C/C=C/c1ccccc1)C(C)O</smiles><smiles>CC(O)C1C=C(C=Cc2ccccc2)COOC1</smiles><smiles>CC(O)C1CC(/C=C/c2ccccc2)OO1</smiles>

38<smiles>CC(O)/C=C/C=C/C(O[10CH3])c1ccccc1</smiles><smiles>CC(O)/C=C/C1COOC1c1ccccc1</smiles>

34<smiles>CC(O)/C=C/C1CC(c2ccccc2)OO1</smiles>

35<smiles>CC(O)/C=C/C(/C=C/c1ccccc1)OO</smiles>

Scheme 4. Suggested mechanism for autoxidation of alcohol 1

\section{Conclusions}

The rich electron substrate allylic hydroxyl system 1 is particularly sensitive towards undergoing an auto-oxidation surprisingly rapidly at $\mathrm{rt}$ in atmospheric air. Several possible, related, mechanistic proposals to explain this auto-oxidation can be forumalted, all of which are initiated due to 1 acting as a sensitizer, reacting directly with dioxygen, and undergoing the alternative decomposition pathways.

\section{Acknowledgments}

We thank NMR and MS services at Durham University.

\section{Experimental Section}

\subsection{General Remarks}

Drying was carried out over anhydrous $\mathrm{MgSO}_{4}$, followed by filtration. Evaporations were carried out at $20 \mathrm{mmHg}$ using a rotary evaporator and water bath. Melting points are uncorrected. TLC was performed on plastic backed silica gel plates or aluminium oxide PET backed plates. All ${ }^{1} \mathrm{H}$ and ${ }^{13} \mathrm{C}$ NMR were recorded using either 400 or $500 \mathrm{MHz}$ spectrometers (for ${ }^{1} \mathrm{H}$ frequencies). All chemicals were purchased from standard chemical suppliers.

\section{$( \pm)-(E)-4-\left(t\right.$-Butyldimethylsilyloxy)-1-iodopent-1-ene $3^{7}$}<smiles>CC(C/C=C/I)O[R15](=S)(=S)c1ccccc1</smiles>

To a solution of $( \pm)-(E)-2-[4-($ tert-butyldimethylsiloxy)-1-pentenyl]-4,4,6-trimethyl-1,3,2dioxaborolane $(1.00 \mathrm{~g}, 3.06 \mathrm{mmol})$ in THF $(10 \mathrm{~mL})$ under Ar at room temperature, was added sodium hydroxide $(1.84 \mathrm{~mL}, 9.19 \mathrm{mmol}, 5 \mathrm{M}$ in de-ionized water). The reaction mixture left to stir 
at $\mathrm{rt}$ for 10 minutes then a solution of iodine $(1.55 \mathrm{~g}, 6.13 \mathrm{mmol})$ dissolved in THF $(5 \mathrm{~mL})$ was added dropwise over ca. 1 minute. The reaction mixture was left to stir at room temperature for $4 \mathrm{~h}$ in the absence of light, followed by the quenching with $5 \%$ aqueous sodium metabisulfite solution $(40 \mathrm{~mL})$. The aqueous layer was extracted with diethyl ether $(3 \times 25 \mathrm{~mL})$, the combined organic extracts were washed with $5 \%$ aqueous sodium metabisulfite solution $(40 \mathrm{~mL})$, water $(40 \mathrm{~mL})$, brine $(40 \mathrm{~mL})$, dried $\left(\mathrm{MgSO}_{4}\right)$, filtered and concentrated. The crude product was purified by silica gel chromatography (petroleum ether as eluent), to give $( \pm)-(E)-4$-(tert-butyldimethylsilyloxy)-1iodopent-1-ene $3(0.714 \mathrm{~g}, 71 \%)$ as a light sensitive yellow oil $(E$-isomeric purity $>99 \%) ; R_{f} 0.83$ (hexane : EtOAc, 4:1); $\lambda_{\max } / \mathrm{cm}^{-1}$ (film): 2928 (w, =C-H), 1472 (w, C=C), 834 (vs), 773 (vs); ${ }^{1} \mathrm{H}$ NMR $\left(400 \mathrm{MHz}, \mathrm{CDCl}_{3}, 20{ }^{\circ} \mathrm{C}\right): \delta_{\mathrm{H}} 0.04(6 \mathrm{H}, \mathrm{s}, \mathrm{MeSi}), 0.88\left(9 \mathrm{H}, \mathrm{s}, \mathrm{Me}_{3} \mathrm{CSi}\right), 1.12(3 \mathrm{H}, \mathrm{d}$, $J 6.0 \mathrm{~Hz}, \mathrm{MeCHOSi}), 2.13-2.22(2 \mathrm{H}, \mathrm{m}, \mathrm{CH}), 3.84(1 \mathrm{H}, \mathrm{h}, J 6.1 \mathrm{~Hz}, \mathrm{CHOSi}), 6.02(1 \mathrm{H}, \mathrm{dt}, J 14.4$, $1.3 \mathrm{~Hz},-\mathrm{CH}=), 6.51(1 \mathrm{H}, \mathrm{dt}, J, 14.4,7.6 \mathrm{~Hz},=\mathrm{CHI}) ;{ }^{13} \mathrm{C} \mathrm{NMR}\left(101 \mathrm{MHz}, \mathrm{CDCl}_{3}\right): \delta_{\mathrm{c}}-4.8(\mathrm{MeSi})$, -4.5 (MeSi), 18.1 (Me $\left.{ }_{3} \mathrm{CSi}\right), 23.5$ (MeCHOSi), 25.84 (Me $\left.{ }_{3} \mathrm{CSi}\right), 46.0\left(\mathrm{CH}_{2}\right), 67.5$ (CHOSi), $128.4(=C H), 143.6(=C H)$; LRMS $\left(\mathrm{ES}^{+}\right): m / z$ (rel. int.) $269.3\left(\mathrm{M}-{ }^{\mathrm{t}} \mathrm{Bu}, 100 \%\right)$; LRMS $(\mathrm{EI}): m / z($ rel. int.) 324.9 (M-H, 21\%), 310.9 (M-I, 100\%); HRMS (TOF ES ${ }^{+}$): Acc. MS calc. for $\mathrm{C}_{11} \mathrm{H}_{22} \mathrm{OSiI}^{+}$[M$\mathrm{H}]^{+}, 325.0485$; found 325.0476 .

\section{( \pm ) 2-(2E, 3E)-[6-(tert-Butyldimethylsiloxy)-1,3-heptadienyl]-4,4,5,5-tetramethyl-1,3,2- dioxaborolane $4^{5 \mathrm{c}}$}

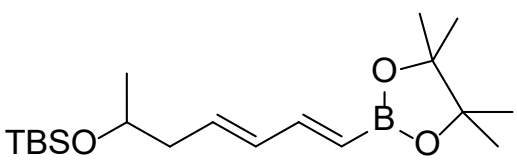

To a dried Schlenk tube under a positive pressure of argon was added $\mathrm{Pd}(\mathrm{OAc})_{2}(120 \mathrm{mg}$, $0.53 \mathrm{mmol})$, silver(I) acetate $(1.92 \mathrm{~g}, 11.8 \mathrm{mmol})$, tri $(o$-tolyl)phosphine $(325 \mathrm{mg}, 1.0 \mathrm{mmol})$, and dry $\mathrm{MeCN}(25 \mathrm{~mL})$. The mixture degassed using the freeze-pump-thaw method $(2 \mathrm{x})$, and vinylboronate pinacol ester $2(1.97 \mathrm{~g}, 12.3 \mathrm{mmol})$ and $(E)$-alkenyl iodide 3 (3.49 $\mathrm{g}, 10.7 \mathrm{mmol})$ were added, and then the mixture was degassed using the freeze-pump-thaw method $(2 \mathrm{x})$ and heated to $50{ }^{\circ} \mathrm{C}$ with vigorous stirring. After $48 \mathrm{~h}$, the mixture was cooled, diluted with $\mathrm{Et}_{2} \mathrm{O}$ (50 $\mathrm{mL})$, passed through Celite, washed with $5 \% \mathrm{HCl}(20 \mathrm{~mL})$, water $(20 \mathrm{~mL})$, and brine $(20 \mathrm{~mL})$, dried $\left(\mathrm{MgSO}_{4}\right)$, and evaporated to give crude product as a yellow oil. Purification by $\mathrm{SiO}_{2}$ chromatography (EtOAc : petroleum ether, 1:19, as eluent) gave the desired product 4 as pale yellow oil (33.4 g, 91\%); $R_{f} 0.64$ (hexane: EtOAc, 4:1); UV $\lambda_{\max }{ }^{\mathrm{EtOH}} \mathrm{nm}(\log \varepsilon) 203$ (4.032), 244 (4.206), 276 (3.677); $\lambda_{\max } / \mathrm{cm}^{-1}$ (film) 2927 (w, C-H), 1604 (s, C=C), 1359 (s), 1255 (s), 1144 (s), 1005 (vs), 833 (vs), 772 (vs); ${ }^{1} \mathrm{H}$ NMR (400 MHz, CDCl 3 ) $\delta_{\mathrm{H}} 0.03$ (3H, s, MeSi), $0.04(3 \mathrm{H}, \mathrm{s}$, $\mathrm{MeSi}), 0.87\left(9 \mathrm{H}, \mathrm{s}, \mathrm{Me} \mathrm{C}_{3} \mathrm{CSi}\right), 1.11(3 \mathrm{H}, \mathrm{d}, J 6.1 \mathrm{~Hz}, \mathrm{MeCHOSi}), 1.27$ (12H, s, $\mathrm{Me}_{2} \mathrm{CCMe}$ ), $2.22(2 \mathrm{H}, \mathrm{m}, \mathrm{CH}), 3.84(1 \mathrm{H}, \mathrm{q}, J 6.0 \mathrm{~Hz}, \mathrm{CHOSi}), 5.43(1 \mathrm{H}, \mathrm{d}, J 17.7 \mathrm{~Hz},=\mathrm{CHB}), 5.88(1 \mathrm{H}, \mathrm{dt}$, $\left.15.3,7.5 \mathrm{~Hz},=\mathrm{CHCH}_{2}\right), 6.14(1 \mathrm{H}, \mathrm{ddd}, J 15.3,10.4,0.8 \mathrm{~Hz}, \mathrm{CH}=\mathrm{CH}-\mathrm{CH}=), 6.98(1 \mathrm{H}, \mathrm{dd}, J 17.7$, $10.4 \mathrm{~Hz},-\mathrm{CH}=\mathrm{CHB}) ;{ }^{13} \mathrm{C} \mathrm{NMR}\left(100 \mathrm{MHz}, \mathrm{CDCl}_{3}\right) \delta_{\mathrm{C}}-4.7(\mathrm{MeSi}),-4.5(\mathrm{MeSi}), 18.1\left(\mathrm{Me}_{3} \mathrm{CSi}\right)$, 23.5 (MeCHOSi), 24.8 (Mes $\mathrm{CSi}), 25.8\left(\mathrm{Me}_{2} \mathrm{CCMe}_{2}\right), 43.1\left(\mathrm{CH}_{2}\right), 68.3(\mathrm{CHOSi}), 83.1(\mathrm{COB})$, $134.4(=C H), 135.8(=C H), 150.1(C H=C H B) ; \delta_{\mathrm{B}}\left(128 \mathrm{MHz}, \mathrm{CDCl}_{3}\right) 30.56 ; \mathrm{LRMS}\left(\mathrm{TOF} \mathrm{AP}^{+}\right): m / z$ (rel. int.) 337.1 (M-Me, 70\%), 221.2 (M-OTBS, 100\%); HRMS (TOF $\mathrm{AP}^{+}$): Acc. MS calc. for $\mathrm{C}_{19} \mathrm{H}_{38}{ }^{10} \mathrm{BO}_{3}{ }^{28} \mathrm{Si}^{+}[\mathrm{M}+\mathrm{H}]^{+}, 352.2720$; found 352.2714.

$((4 E, 6 E)-7-P h e n y l h e p t a-4,6-d i e n-2-y l o x y)\left(\right.$ tert-butyl)dimethylsilane $5^{8}$<smiles>CC(C)OC(C)C/C=C/C=C/c1ccccc1</smiles>

To a dried Schlenk tube under a positive pressure of argon was added iodobenzene (400 $\mathrm{mg}$, $1.961 \mathrm{mmol})$ and dialkenyl boronate $4(698 \mathrm{mg}, 1.980 \mathrm{mmol})$ in DMF $(20 \mathrm{~mL})$ and $\mathrm{H}_{2} \mathrm{O}(0.2 \mathrm{~mL})$. The mixture degassed using the freeze-pump-thaw method $(1 \mathrm{~mL})$, and $\mathrm{Ba}(\mathrm{OH})_{2}(403 \mathrm{mg}$, $2.535 \mathrm{mmol})$ and $\mathrm{Pd}(\mathrm{dppf}) \mathrm{Cl}_{2}(72 \mathrm{mg}, 0.098 \mathrm{mmol})$ were added, and then the mixture was degassed 
using the freeze-pump-thaw method $(3 \mathrm{x})$. The mixture was vigorously stirred at room temperature for $48 \mathrm{~h}$ before it was diluted with EtOAc $(100 \mathrm{~mL})$ and passed through Celite, washed with $5 \%$ $\mathrm{HCl}(25 \mathrm{~mL})$, water $(25 \mathrm{~mL})$, and brine $(25 \mathrm{~mL})$, dried $\left(\mathrm{MgSO}_{4}\right)$, and evaporated to give crude product as a yellow oil. Purification by $\mathrm{SiO}_{2}$ chromatography (EtOAc/hexane, 0.5:9.5 as eluent) gave the desired product 5 as yellow oil (396 mg, 71\%); $\mathrm{R}_{\mathrm{f}}\left(\mathrm{Hex}\right.$ : EtOAc 4:1) 0.78; $v_{\max } / \mathrm{cm}^{-1}$ (film) 2955 (m), 2927 (m), 2855 (m), 1360 (s), 1252 (s), 1080 (s), 987 (vs), 833 (vs), 723 (vs), 690 (s); $\delta_{\mathrm{H}}$ $\left(400 \mathrm{MHz}, \mathrm{CDCl}_{3}\right) 0.05(3 \mathrm{H}, \mathrm{s}, \mathrm{MeSi}), 0.06(3 \mathrm{H}, \mathrm{s}, \mathrm{MeSi}), 0.89$ (9H, s, Me $\left.3 \mathrm{CSi}\right), 1.12(3 \mathrm{H}, \mathrm{d}$, $J$ 6.1, MeCHOSi), 2.20-2.31 (2H, m, CH 2 CHOSi), 3.83-3.91 (1H, m, CHOSi), 5.77-5.84 (1H, dt, $J$ 15.1, 7.5, $\left.=\mathrm{CHCH}_{2}\right), 6.21\left(1 \mathrm{H}, \mathrm{dd}, J 15.2,9.8, \mathrm{CH}=\mathrm{CHCH}_{2}\right), 6.45(1 \mathrm{H}, \mathrm{d}, J 15.7, \mathrm{PhCH}=\mathrm{CH})$, $6.75(1 \mathrm{H}, \mathrm{dd}, J 15.7,10.2, \mathrm{PhCH}=\mathrm{C} H), 7.17-7.22\left(1 \mathrm{H}, \mathrm{m}, H-4^{\prime}\right), 7.28-7.32\left(2 \mathrm{H}, \mathrm{m}, H-3^{\prime} \& H-5^{\prime}\right)$, 7.37-7.39 (2H, m, H-2' \& H-6' ); $\delta_{\mathrm{C}}\left(100 \mathrm{MHz}, \mathrm{CDCl}_{3}\right)-4.65$ (MeSi), -4.52 (MeSi), 18.17 (Me $\left.{ }_{3} \mathrm{CSi}\right), 23.58$ (MeCHOSi), $25.88\left(\mathrm{Me}_{3} \mathrm{CSi}\right), 43.24\left(\mathrm{CH}_{2}\right), 68.63(\mathrm{CHOSi}), 126.16\left(\mathrm{C}-2^{\prime}\right.$ \& $\left.C-6^{\prime}\right), 127.13(\mathrm{PhCH}=C \mathrm{H}), 128.53\left(C-3^{\prime} \quad \& \quad C-5^{\prime}\right), 129.31 \quad\left(C \mathrm{H}=\mathrm{CHCH}_{2}\right), 130.40 \quad(\mathrm{PhCH}=)$, $\left.132.11\left(=\mathrm{CHCH}_{2}\right), 137.02\left(C-1^{\prime}\right) ;^{\prime}\right)$; LRMS (CI): $m / z$ (rel. int.) 287.1 (M-Me, 2\%), $248\left(\mathrm{M}-{ }^{\mathrm{t}} \mathrm{Bu}\right.$, 8\%), 171.1 (M-OTBS, 40\%), 159.1 (MeCHOTBS-M, 100\%).

\section{(4E,6E)-7-Phenylhepta-4,6-dien-2-ol $1^{9}$}<smiles>CC(O)CC=CC=Cc1ccccc1</smiles>

The TBSO-ether compound $5(390 \mathrm{mg}, 1.29 \mathrm{mmol})$ was dissolved in THF (15 mL) and TBAF ( $2.6 \mathrm{~mL}, 2.58 \mathrm{mmol}, 1 \mathrm{M}$ solution in THF) was added drop wise at $\mathrm{rt}$. The result mixture is stirred at $\mathrm{rt}$ until the TLC indicated the consumption of starting material. The reaction mixture is diluted with $50 \mathrm{~mL}$ EtOAc, washed with $25 \mathrm{~mL}$ brine, $25 \mathrm{~mL}$ water, dried over $\mathrm{MgSO}_{4}$, concentrated in vacuo and the residue was purified by $\mathrm{SiO}_{2}$ chromatography (EtOAc : petroleum ether, 1:4 as eluent) to give the desired product 1 as yellow solid (180 mg, 74\%); $R_{f} 0.18$ (hexane: EtOAc, 4:1); Mp 48-50 ${ }^{\circ} \mathrm{C}$; UV $\lambda_{\max }{ }^{\mathrm{EtOH}} \mathrm{nm}(\log \varepsilon) 192$ (3.624), 200 (3.563), 228 (3.463), 288 (3.492), 332 (3.563); $v_{\max } / \mathrm{cm}^{-1}$ (film) 3294 (br, OH), 3019 (m), 2963 (m), 2926 (m), 1595 (m), 1447 (m), 1370 (m), 1119 (m), 1066 (s), 992 (vs), 746 (vs), 690 (vs); ${ }^{1} \mathrm{H}$ NMR (400 MHz, CDCl, $20{ }^{\circ} \mathrm{C}$ ): $\delta_{\mathrm{H}} 1.24(3 \mathrm{H}, \mathrm{d}, J 6.2, \mathrm{MeCHOH}), 2.23-2.38\left(2 \mathrm{H}, \mathrm{m}, \mathrm{CH}_{2} \mathrm{CHOH}\right), 3.85-3.93(1 \mathrm{H}, \mathrm{m}, \mathrm{CHOH}), 5.82$ $\left(1 \mathrm{H}, \mathrm{dt}, J 15.1,7.6,=\mathrm{CHCH}_{2}\right), 6.30\left(1 \mathrm{H}, \mathrm{ddd}, J 15.1,10.4,0.6, \mathrm{C} H=\mathrm{CHCH}_{2}\right), 6.49(1 \mathrm{H}, \mathrm{d}, J 15.7$, $\mathrm{PhCH}=\mathrm{CH}), 6.77(1 \mathrm{H}, \mathrm{dd}, J 15.7,10.4, \mathrm{PhCH}=\mathrm{CH}), 7.17-7.23\left(1 \mathrm{H}, \mathrm{m}, H-4^{\prime}\right), 7.28-7.32(2 \mathrm{H}, \mathrm{m}, H-$ $\left.3^{\prime} \& H-5^{\prime}\right)$, 7.37-7.39 (2H, m, H-2' \& H-6 $)$; ${ }^{13} \mathrm{C} \mathrm{NMR}\left(101 \mathrm{MHz}, \mathrm{CDCl}_{3}\right): \delta_{\mathrm{c}} 22.9(\mathrm{MeCHOH})$, $42.8\left(\mathrm{CH}_{2}\right), 67.4(\mathrm{CHOH}), 126.2\left(C-2^{\prime} \quad \& C-6^{\prime}\right), 127.4(\mathrm{PhCH}=\mathrm{CH}), 128.6\left(C-3^{\prime} \& C-5^{\prime}\right), 128.8$ $\left(\mathrm{CH}=\mathrm{CHCH}_{2}\right), 130.6(\mathrm{PhCH}=), 131.3\left(=\mathrm{CHCH}_{2}\right), 133.8\left(C-1^{\prime}\right)$; LRMS (CI): $m / z$ (rel. int.) 188.2 $\left(\mathrm{M}^{+}, 30 \%\right), 171.1(\mathrm{M}-\mathrm{OH}, 90 \%), 129.0(100 \%), 91 \%\left(\mathrm{PhCH}_{2}-\mathrm{M}, 56 \%\right)$; HRMS (TOF AP $\left.{ }^{+}\right)$: Acc. MS calc. for calcd for $\mathrm{C}_{13} \mathrm{H}_{15} \mathrm{O}^{+}[\mathrm{M}-\mathrm{H}]^{+}, 187.1123$; found 187.1112 .

\section{References}

[1] M. G. Simic, J. Chem. Educ. 1981, 58, 125-131.

[2] P. E. Correa, G. Hardy, D. P. Riley, J. Org. Chem. 1988, 53, 1695-1702.

[3] J. H. Lee, Photooxidation and photosensitized oxidation in linoleic acid, milk, and lard. $\mathrm{PhD}$ thesis, 2002, The Ohio State University, Columbus, OH, USA.

[4] E. N. Frankel, Prog. Lipid Res. 1980, 19, 1-22.

[5] a) G. N. Maw, C. Thirsk, J.-L. Toujas, M. Vaultier, A. Whiting, Synlett, 2004, 1183-1186; b) Batsanov, A. S.; Knowles, J. P.; Whiting, A. J. Org. Chem. 2007, 72, 2525-2532; c) A. S. Batsanov, J. P. Knowles, A. P. Lightfoot, G. Maw, C. E. Thirsk, S. J. R. Twiddle, A. Whiting, Org. Lett., 2007, 9, 5565-5568; d) J. P. Knowles, V. E. O’Connor, A. Whiting, Org. Biomol. Chem., 2011, 9, 1876-1886 
[6] a) A. R. Hunt, S. K. Stewart, A. Whiting, Tetrahedron Lett., 1993, 34, 3599-3602; b) S. K. Stewart, A. Whiting, J. Organomet. Chem., 1994, 482, 293-300; c) S. K. Stewart, A. Whiting, Tetrahedron Lett., 1995, 36, 3925-392.

[7] C. Morrill, R. H. Grubbs, J. Org. Chem. 2003, 68, 6031-6034.

[8] N. Jiang, A. J. Ragauskas, Tetrahedron Lett. 2006, 47, 197-200.

[9] T. W Greene, P. G. M Wuts, Protective Groups in Organic Synthesis, 3rd ed.; 1999, John Wiley \& Sons: New York. 